\title{
Finite Groups of Derangements on the $n$-Cube II
}

\author{
Larry Cusick \\ Department of Mathematics \\ California State University, Fresno. \\ Fresno, California, USA \\ larryc@csufresno.edu
}

\author{
Oscar Vega * \\ Department of Mathematics \\ California State University, Fresno. \\ Fresno, California, USA \\ ovega@csufresno.edu
}

Submitted: Mar 23, 2011; Accepted: Sep 20, 2011; Published: Oct 3, 2011

Mathematics Subject Classification: Primary 05E18; Secondary 05E10, 52C99, $20 B 25$

\begin{abstract}
Given $k \in \mathbb{N}$ and a finite group $G$, it is shown that $G$ is isomorphic to a subgroup of the group of symmetries of some $n$-cube in such a way that $G$ acts freely on the set of $k$-faces, if and only if, $\operatorname{gcd}(k,|G|)=2^{s}$ for some non-negative integer $s$. The proof of this result is existential but does give some ideas on what $n$ could be.
\end{abstract}

\section{Preliminaries}

The $n$-dimensional cube, or simply $n$-cube, is denoted by $Q_{n}$ and will be represented as having vertices the points of $\{1,-1\}^{n} \subset \mathbb{R}^{n}$, and edges joining any two vertices that differ in exactly one component. A $k$-face $F$ of the $n$-cube is a $k$-subcube whose vertices have $n-k$ of the coordinates predetermined,

$$
F=\left\{\mathbf{y}=\left(y_{1}, \ldots, y_{n}\right) \in Q_{n} ; y_{i_{1}}=a_{i_{1}}, \ldots, y_{i_{n-k}}=a_{i_{n-k}}\right\},
$$

where, of course, each $a_{i_{j}}= \pm 1$.

It is known that the automorphism group of the cube is $B_{n}=S_{n} 2 \mathbb{Z}_{2}$, the wreath product of $S_{n}$ and $\mathbb{Z}_{2}$ (in this article we will use $\mathbb{Z}_{2}=\{ \pm 1\}$ ). This group is sometimes called the hyperoctahedral group, or the group of signed permutations; it is a Coxeter group of type $B_{n}=C_{n}$, and thus a Weyl group. We denote the elements in $B_{n}$ by $(\sigma ; \mathbf{x})$, where $\sigma \in S_{n}$ and $\mathbf{x}=\left(x_{1}, x_{2}, \cdots, x_{n}\right) \in\left(\mathbb{Z}_{2}\right)^{n}$. The multiplication is given by

$$
(\sigma ; \mathbf{x})(\tau ; \mathbf{y})=\left(\sigma \tau ; \mathbf{x}^{\tau} \mathbf{y}\right)
$$

where $\mathbf{x}^{\tau}=\left(x_{\tau(1)}, x_{\tau(2)}, \cdots, x_{\tau(n)}\right)$, and $\mathbf{x}^{\tau} \mathbf{y}$ is the standard component-to-component multiplication in $\mathbb{R}^{n}$. The (right) action of $B_{n}$ on $Q_{n}$ is given by $(\sigma, \mathbf{x}) \mathbf{y}=\mathbf{y}^{\sigma} \mathbf{x}$.

*The authors would like to thank the referees for their helpful advice, which greatly improved the exposition. 
Definition 1. With the same notation as above.

1. Let $G$ be a group acting on a set $X$. We say that $g \in G$ acts freely on $X$ if and only if $g$ does not fix any points in $X$.

2. A derangement of the $k$-faces of $Q_{n}$ is an element of $B_{n}$ that acts freely on the set of all $k$-faces of $Q_{n}$.

3. A subgroup $H$ of $B_{n}$ is said to be a derangement of the $k$-faces of $Q_{n}$ if every non-identity element in $H$ is a derangement of the $k$-faces of $Q_{n}$.

4. A group $G$ will be called a derangement of the $k$-faces of $Q_{n}$ if it is isomorphic to subgroup of $B_{n}$ that is a derangement of the $k$-faces of $Q_{n}$. In such a case we introduce the notation

$$
G \vdash_{k} B_{n} .
$$

We want to study conditions for a finite group $G$ to be a derangement of the $k$-faces of some $Q_{n}$. The main tool we will use in this article is the Chen-Stanley criterion. In order to get to it we first need to set some notation.

Definition 2. If $\sigma=\left(i_{1}, i_{2}, \ldots, i_{s}\right)$ is a cycle in $S_{n}$ and $\mathbf{x} \in\left(\mathbb{Z}_{2}\right)^{n}$, then

$$
x_{\sigma}=x_{i_{1}} x_{i_{2}} \cdots x_{i_{s}} .
$$

Theorem 1. (Chen-Stanley Criterion [2]) A symmetry $(\pi ; \mathbf{x}) \in B_{n}$ is a derangement of the set of $k$-faces in $Q_{n}$ if, and only if, for every $k$-element $\pi$-invariant subset $I \subset$ $\{1, \ldots, n\}, x_{\sigma}=-1$ for some cycle $\sigma$ in $\pi$ disjoint from $I$.

Note that, in particular, $(\pi ; \mathbf{x}) \in B_{n}$ is a vertex-derangement (i.e. $k=0$ ) if, and only if, $x_{\sigma}=-1$ for some cycle $\sigma$ in $\pi$. This is because there is one zero-element subset (the empty set), which is pi-invariant (vacuously) and every cycle is disjoint from the empty set.

In a previous article [3], the first author proved the following results.

Theorem 2. Assume $k$ and $n$ are always non-negative integers, and that the notation is the same used before

(i) If $G$ is a group of odd order, then $G \vdash_{k} B_{n}$ for some $n$ if, and only if, $\operatorname{gcd}(k,|G|)=1$.

(ii) For any $m \geq 2$ and $k \geq 0, \mathbb{Z}_{m} \vdash_{k} B_{n}$ for some $n$ if, and only if, $\operatorname{gcd}(k, m)=2^{s}$ for some $s \geq 0$.

(iii) If $G$ is a finite group and $G \vdash_{k} B_{n}$ for some $n \geq 1$, then $\operatorname{gcd}(k,|G|)=2^{\text {s }}$ for some $s \geq 0$.

(iv) If $|G|=2^{s}$, then for all $k$ there exists an $n$ such that $G \vdash_{k} B_{n}$.

The main theorem in this article (theorem 6) is, essentially, the converse of theorem 2 (iii). We now move on to present concepts and results that will be needed in the proof of theorem 6 . 


\section{Sufficiency}

We can think of $G \vdash_{k} B_{n}$ as saying there is a faithful representation of $G$ in the group of signed permutations, with an extra condition. Also, the hyperoctahedral group contains a copy of $S_{n}$, so any faithful representation of a group $G$ into $S_{n}$ can be easily 'extended' to an injective homomorphism $G \rightarrow B_{n}$.

Definition 3. With the same notation used in the previous section we define:

1. An element $(\pi ; \mathbf{x}) \in B_{n}$ is called sufficient if the following condition is satisfied.

(a) If $(\pi ; \mathbf{x})$ is of odd order, then $\pi$ has no fixed points.

(b) If $(\pi ; \mathbf{x})$ is of even order, then there is a cycle $\sigma$ in $\pi$ for which $x_{\sigma}=-1$.

2. A representation of a group $G$ into $B_{n}$ is a homomorphism $\rho: G \rightarrow B_{n}$.

3. A representation $\rho: G \rightarrow B_{n}$ is called sufficient if $\rho(g)$ is sufficient for every nonidentity element $g \in G$.

Our idea is to consider a sufficient representation of a group $G$ and then 'multiply' it with itself to create a representation for $G$ that satisfies the conditions of the Chen-Stanley criterion. The way of multiplying representations we will use is defined next.

Definition 4. The outer product $\times: B_{n} \times B_{m} \rightarrow B_{n+m}$ is defined by

$$
(\pi ; \mathbf{x}) \times(\theta ; \mathbf{y})=(\pi \times \theta ; \mathbf{x}, \mathbf{y})
$$

where $\pi \times \theta$ is the permutation given by

$$
\pi \times \theta=\left(\begin{array}{ccccccc}
1 & 2 & \cdots & n & n+1 & \cdots & n+m \\
\pi(1) & \pi(2) & \cdots & \pi(n) & n+\theta(1) & \cdots & n+\theta(m)
\end{array}\right)
$$

The following fundamental construction will allow us to link the concepts of sufficient representation and derangements of $k$-faces.

Remark 1 (Fundamental Construction) Let $\Delta_{t}, \Delta_{t}^{(i)}: B_{n} \rightarrow B_{n t}$ be given by $\Delta_{t}(g)=$ $\underbrace{g \times \cdots \times g}_{t \text { times }}$ and $\Delta_{t}^{(i)}(g)=\underbrace{1 \times \cdots \times g \times \cdots \times 1}_{t \text { factors }}$, where the element $g$ appears only in the $i$-position. Note that $\Delta_{t}(g)=\Delta_{t}^{(1)}(g) \cdots \Delta_{t}^{(n)}(g)$.

For a cycle $\sigma=\left(i_{1}, \ldots, i_{r}\right)$, let $\tilde{\sigma}$ be the set $\left\{i_{1}, \ldots, i_{r}\right\}$, and for a permutation $\pi$ of $\{1, \ldots, n\}$ with cycle decomposition $\pi=\sigma_{1} \cdots \sigma_{\ell}$, let the cycle set of $\pi$ be the set $\left\{\tilde{\sigma}_{1}, \ldots, \tilde{\sigma}_{\ell}\right\}$.

Now notice that if we write $\Delta_{t}(g)=(\theta, \mathbf{y})$ and $\Delta_{t}^{(i)}(g)=\left(\theta^{(i)}, \mathbf{y}^{(i)}\right)$, then the cycle set for $\theta$ is equal to the disjoint union $S=S_{1} \cup \cdots \cup S_{n}$ where each $S_{i}$ is the cycle set for $\theta^{(i)}$. It follows that for any fixed natural number $k$, and $g \in B_{n}$, there is a sufficiently large natural number $t(t>k$ will do) so that any $k$-element subset $I \subset\{1, \ldots, n t\}$ is disjoint from some cycle set $S_{i}$ as derived from $\Delta_{t}^{(i)}(g)$ above. 
Theorem 3. Suppose $\operatorname{gcd}(|G|, k)=2^{s}$ for some $s$, and there is a sufficient representation $\rho: G \rightarrow B_{r}$. Then $G \vdash_{k} B_{q}$ for some $q$.

Proof. First, suppose $g=(\pi, \mathbf{x}) \in B_{r}$ is an even order element and $x_{\sigma}=-1$ for some cycle $\sigma$ in $\pi$. Then, by the Fundamental Construction above, there is a sufficiently large outer product $\Delta_{t}(g)=(\theta, \mathbf{y})$ for which if $I \subset\{1, \ldots, r t\}(\theta$-invariant or not) then $I$ is disjoint from some cycle set $S_{i}$. By assumption, $x_{\sigma}=-1$. The corresponding equivalent cycle $\sigma^{\prime}$ in $\theta^{(i)}$, hence in $\theta$, then satisfies $y_{\sigma^{\prime}}=x_{\sigma}=-1$.

Now suppose $g=(\pi, \mathbf{x})$ is non-trivial and has odd order, $\pi$ has no fixed points, and $\operatorname{gcd}(|g|, k)=2^{s}$ for some $s$. Then, by necessity, $\operatorname{gcd}(|g|, k)=1$. Let $\Delta_{t}(g)=(\theta, \mathbf{y})$. It also follows that $\theta$ is an odd order permutation, and so for any $t$ there is no $k$-element $\theta$-invariant subset $I \subset\{1, \ldots, r t\}$.

Now, we may assume $G<B_{r}$ and $\operatorname{gcd}(|G|, k)=2^{s}$ for some $s$. By choosing $t$ to be sufficiently large for all even order elements, we have a representation $\rho: G \rightarrow B_{r t}$ that satisfies the Chen-Stanley condition.

What is now left to be proved is that every group $G$ such that $\operatorname{gcd}(|G|, k)=2^{s}$, for some $s$, admits a sufficient representation in some $B_{n}$. We will prove this in the next section by inducing a representation for $G$ from its 2-Sylow subgroup (recall that the case $|G|$ odd has already been discussed in theorem 2). The following theorem justifies us wanting to induce from the 2-Sylow subgroup of $G$.

Theorem 4 (See [3]). Every finite 2-group has a sufficient representation.

\section{Induced Representations}

Suppose $H$ is a subgroup of a finite group $G$ of index $m$ and $\rho_{0}: H \rightarrow B_{n}$ is a faithful representation. There is a representation $\rho: G \rightarrow B_{n m}$, induced up from $\rho_{0}$ whose construction we will now describe.

First choose a complete set of coset representatives $\left\{g_{1}, \ldots, g_{m}\right\}$ of the subgroup $H$,

$$
G=g_{1} H \cup \cdots \cup g_{m} H
$$

Pick $g \in G$. For each $i=1, \ldots, m$, the product $g g_{i}$ is in one of the cosets, and so $g g_{i}=g_{\theta(i)} h_{i}$ for some permutation $\theta$ of $\{1, \ldots, m\}$ and $h_{i} \in H$. We can write each $\rho_{0}\left(h_{i}\right)=\left(\pi_{i} ; \mathbf{x}_{i}\right)$. Then

$$
\rho(g)=\left(\pi ; \mathbf{x}_{\mathbf{1}}, \ldots, \mathbf{x}_{m}\right)
$$

where $\pi$ is the permutation on $\{1, \ldots, n m\}$ that permutes the successive $m$-blocks via $\theta$, while the block interiors are permuted via the corresponding $\pi_{i}$. Specifically, for $j \in$ $\{1, \ldots, n m\}$, write $j=a n+b$ where $0 \leq a<m$ and $0<b \leq n$, then

$$
\pi(j)=\pi_{\theta(a+1)}(b)+(\theta(a+1)-1) n .
$$


Remark 2 Note that if we restrict the induced representation $\rho$ back to the subgroup $H$, then $\left.\rho\right|_{H}$ is the direct sum of $m$ copies of $\rho_{0}$ (see, for example, [5]). Thus, for $h \in H$,

$$
\rho(h)=\rho_{0}(h) \times \cdots \times \rho_{0}(h) \quad(m \text { times }) .
$$

It follows immediately that if $\rho_{0}$ is sufficient, then so is $\left.\rho\right|_{H}$.

Lemma 1. If $H$ is a finite 2-group, $g \in G$ is an odd order element and $\rho(g)=(\pi ; \mathbf{x})$, then $\pi$ has no 1-cycle. That is, $\rho(g)$ is sufficient.

Proof. Suppose $\pi$ has a 1-cycle. Then $\theta$ must fix one block, that is $\theta$ has a 1 -cycle. So, $g g_{j}=g_{j} h$ for some $j=1, \ldots, m$ and $h \in H$. Thus, $g_{j}^{-1} g g_{j} \in H$, that is $g$ cannot be of odd order.

Theorem 5 (See [3]). Two symmetries $(\theta ; \mathbf{y}),(\pi ; \mathbf{x}) \in B_{n}$ are conjugate if, and only if,

(1) $\theta$ and $\pi$ have the same cycle structure and

(2) for some pairing of respectively equal length cycles in the two permutations $\tau_{1} \longleftrightarrow$ $\sigma_{1}, \ldots, \tau_{s} \longleftrightarrow \sigma_{s}$, we have $y_{\tau_{j}}=x_{\sigma_{j}}$ for all $j=1, \ldots, s$.

Corollary 1. If $H$ is a Sylow 2-subgroup of $G, \rho_{0}$ is sufficient, and $g \in G$ is an element whose order is a power of 2 , then $\rho(g)$ is sufficient.

Proof. Since Sylow subgroups are conjugate, some conjugate of $g$ is an element of $H$. The corollary now follows from theorem 5 , the assumptions and remark 2 .

\section{Main Theorem}

It is our aim in this section to prove:

Theorem 6 (Main Theorem). Suppose $G$ is a finite group and $k$ is a non-negative integer with $\operatorname{gcd}(|G|, k)=2^{s}$ for some non-negative integer $s$, then there is positive integer $q$ for which $G \vdash_{k} B_{q}$.

According to Theorem 3, the Main Theorem will follow from the assumptions if we can prove the existence of a sufficient representation $\rho: G \rightarrow B_{r}$ for some $r$.

Theorem 7. Every finite group has a sufficient representation.

We begin with a few lemmas.

Lemma 2. In $B_{m}$, Suppose $\alpha=(\sigma ; \mathbf{x})$ where $\sigma=(12 \ldots m)$ and $\alpha^{t}=\left(\sigma^{t} ; \mathbf{y}\right)$. Then $y_{\sigma}=\left(x_{\sigma}\right)^{t / \operatorname{gcd}(m, t)}$.

Proof. The permutation $\sigma^{t}$ is a product of $(\mathrm{m} / \operatorname{gcd}(m, t))$-cycles in the form $(i, i+t, \ldots, i+$ $(m / \operatorname{gcd}(m, t)-1) t)$ for $i=1, \ldots, \operatorname{gcd}(m, t)$ where terms are $\bmod m$. And, the $j$ th component of $\mathbf{y}$ is $y_{j}=x_{j} x_{j+1} \cdots x_{j+t-1}$ (indices computed $\bmod m$ ). Thus,

$$
\begin{aligned}
y_{\sigma} & =\left(x_{1} \cdots x_{i+t-1}\right) \cdots\left(x_{i+(m / \operatorname{gcd}(m, t)-1) t}, \ldots x_{i+m t / \operatorname{gcd}(m, t)-1}\right) \\
& =x_{1} x_{2} \ldots x_{m t / \operatorname{gcd}(m, t) \quad(\text { indices } \bmod m)} \\
& =\left(x_{\sigma}\right)^{t / \operatorname{gcd}(m, t)} .
\end{aligned}
$$


Remark 3 It is known that any element $\alpha \in B_{m}$ is a product of disjoint bicycles. A bicycle is any element $(\sigma ; \mathbf{x}) \in B_{m}$ in which $\sigma$ is a cycle and $x_{j}=1$ if $\sigma(j)=j$. Two bicycles are called disjoint if their respective permutation parts are disjoint in the usual sense. See [3] for more details.

Lemma 3. Suppose $\alpha=(\pi ; \mathbf{x}) \in B_{m}$ and $x_{\sigma}=1$ for every cycle $\sigma$ in $\pi$. If $\alpha^{t}=\left(\pi^{t} ; \mathbf{y}\right)$, then $y_{\psi}=1$ for every cycle $\psi$ in $\pi^{t}$.

Proof. By factoring $\alpha$ as a product of disjoint bicycles, it is enough to prove the lemma for $\pi=$ cycle. And, in fact, we may assume $\alpha=(\sigma ; \mathbf{x}) \in B_{m}$ where $\sigma$ is the cycle $(12 \ldots m)$, as external products will allow us to 'paste' these cycles. Lemma 3 now follows from lemma 2.

We can now prove the Main Theorem.

Proof of Theorem 7. Let $H$ be a Sylow 2-subgroup of $G$, of index $m$. By corollary 4, there is a sufficient representation $\rho_{0}: H \rightarrow B_{n}$ for some $n$. Let $\rho: G \rightarrow B_{n m}$ be the representation induced up from $\rho_{0}$. We will prove $\rho$ is sufficient.

Pick $g \in G$, a non-identity element. If the order of $g$ is odd or a power of 2 , then $\rho(g)$ is sufficient by lemma 1 and corollary 1 . Now assume the order of $g$ to be $2^{a}(2 b+1)$ with

$a>0$. Notre that $g^{\prime}=g^{2 b+1}$ has order $2^{a}$, and so $\rho\left(g^{\prime}\right)$ is sufficient. It follows that if we write $\rho\left(g^{\prime}\right)=(\pi ; \mathbf{x})$, then $x_{\sigma}=-1$ for some cycle $\sigma$ in $\pi$. It follows from lemma 3 , that if $\rho(g)=(\theta ; \mathbf{y})$, then $y_{\psi}=-1$ for some cycle $\psi$ in $\theta$. That is, $\rho(g)$ is sufficient.

\section{References}

[1] M. Baake. Structure and Representations of the Hyperoctahedral Group, J. Math. Phys. 25, (1984), 3171-3182.

[2] W. Y. C. Chen \& R. P. Stanley. Derangements of the n-cube, Discrete Mathematics 115 (1993) 65-75.

[3] L. W. Cusick. Finite Groups of Derangements on the n-Cube, To Appear in Ars Combinatoria.

[4] N. Metropolis \& G-C. Rota. Combinatorial Structure of the Faces of the n-Cube, SIAM J. Appl. Math Vol. 35, No. 4 (1978), 689-694.

[5] J. P. Serre. Linear Representations of Finite Groups, Springer-Verlag (1977). 